\title{
Transforming Photographs into Sculptures
}

\author{
Lee Wagstaff \\ Royal College of Art - Fine art Research \\ Hertzberg Str.27, \\ 12055 Berlin, Germany \\ lee.wagstaff@network.rca.ac.uk
}

\section{INTRODUCTION}

With $3 \mathrm{~d}$ printing still hitting the headlines offering a multitude of miracle claims from organ construction to a manufacturing revolution. It is time to look past the hype and see how this technology can be more widely utilized and further developed in the hands of artists, designers and hobbyists. One of the main problems for $3 \mathrm{~d}$ printing enthusiasts in the past was finding or creating original things to print. Ambitious $3 d$ projects used to require external expert technical assistance and a large budget. To create $3 \mathrm{~d}$ models from scratch requires certain proficiency with computers and a large amount of skill and time. This is where I believe that the process of photogrammetry has a role to play.

\section{DEMONSTRATION}

During my EVA demonstration I will show from beginning to end the process of turning photographs into sculpture using digital photogrammetry.

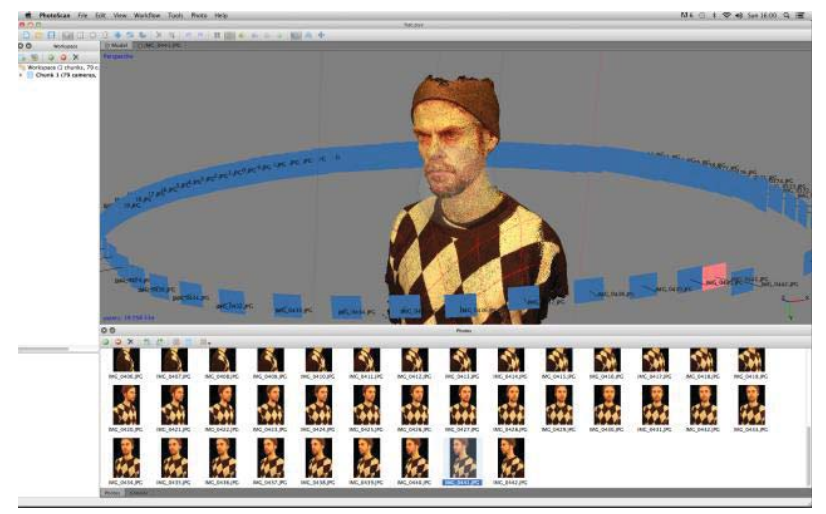

Figure 1: Screen grab of multiple image alignment

The aim of this presentation is to demonstrate the potential of photogrammetry for artists and to inspire them to create new sculptural artworks from digital images. It will explore the simplicity of the process, how to get the best results and the possible applications.

\subsection{Method}

Photogrammetry offers the opportunity to digitize existing objects and the relative simplicity of the technique also allows those with little digital experience to participate and create.

During the demonstration I will take multiple photographs of a subject on a turntable using a regular consumer camera, upload the images to a laptop and process them using software that will create a $3 \mathrm{~d}$ model. I will talk about preparing this model for $3 d$ printing, how the model can be creatively edited and show examples of models I have printed.

Photogrammetry creates very detailed textures, which makes this a perfect technique for the long term archiving of artworks as digital files but also as physical artefacts. As a fine artist I am also interested in photogrammetry as a flexible, creative medium in its own right. Combined with $3 \mathrm{~d}$ printing this process could be used for creating maquettes, models for lost wax casting, and if the model is inverted for printing master moulds.

Modern photogrammetry is a process that uses software to create three-dimensional representations of real world objects from multiple digital photographs of that object. The software is able to align the photographs and construct the model with the bonus that the texture (surface imagery) of the object is also reconstructed. These models can be printed in their pure forms as exact miniature models or manipulated to create new forms. This process is perfect for scanning people, buildings and cultural artefacts in full colour. Photogrammetry has great potential as it can be used in a studio or in the field; it is a non-evasive, non-contact procedure that requires only basic technical skill.

Two great advantage of photogrammetry over other forms of $3 d$ scanning such as laser or fringe pattern scanning is the cost and flexibility. All that is 
required is a digital camera, a turntable, a tripod and a computer with software; this set up is accessible to artists and economically viable.

Although this technique has been available for several decades' recent advances in user-friendly software, computer processing power and digital camera quality has made this process more accessible to artists, designers and hobbyists.

\section{FURTHER READING}

Foster, S \& Halbstein, D. (2014) Integrating 3D Modeling, Photogrammetry and Design. Springer, Rochester NY.

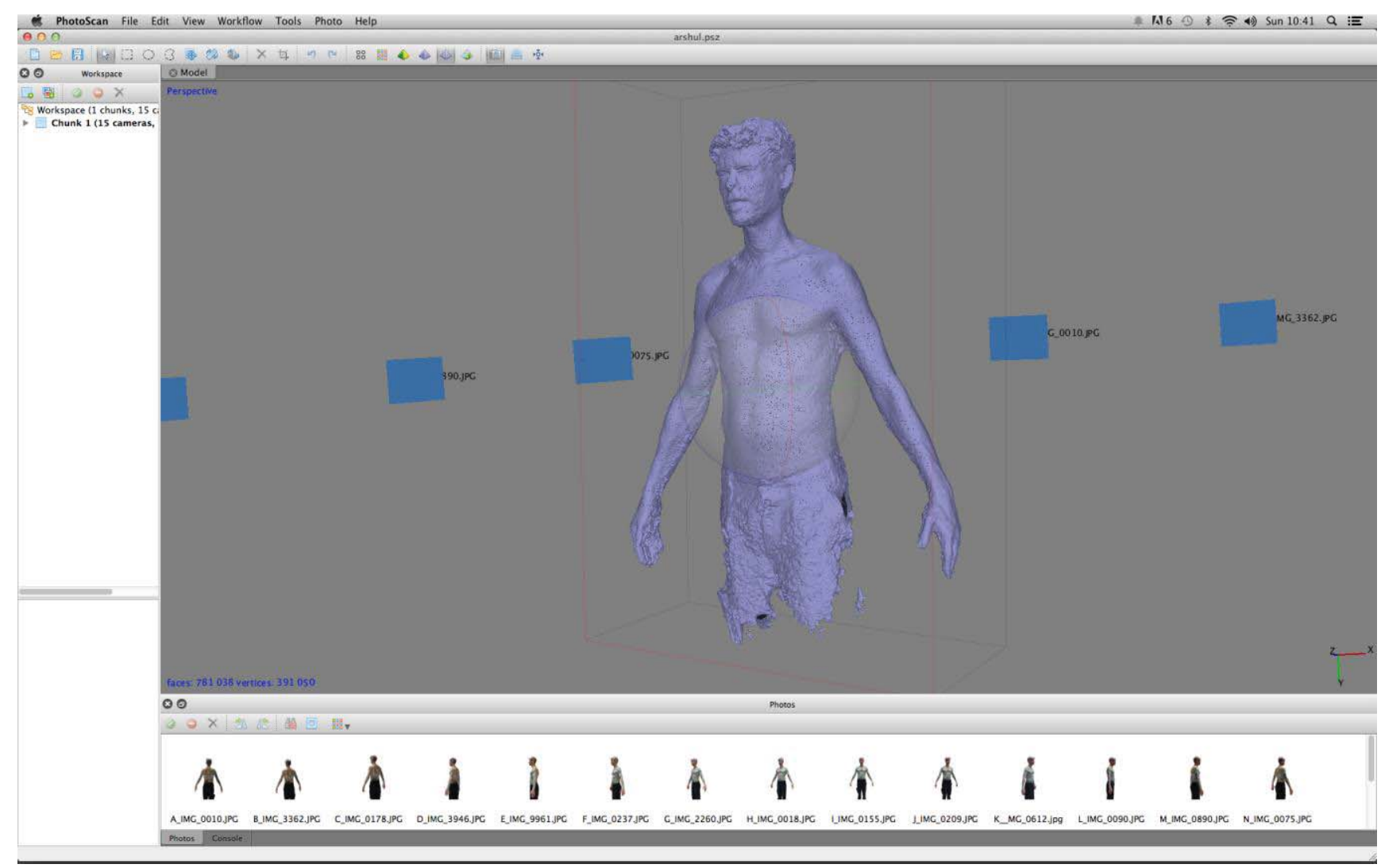

Figure 2: Wire Model. Photogrammetry model generated from 15 images 